\title{
Invited Discussion on: Aesthetic Analysis of Alteration of Eyebrow Position After Double Eyelidplasty
}

\author{
Yiye Ouyang ${ }^{1} \cdot$ Jie Luan $^{1}$ (D)
}

Received: 3 January 2020/Accepted: 5 January 2020/Published online: 22 January 2020

(C) Springer Science+Business Media, LLC, part of Springer Nature and International Society of Aesthetic Plastic Surgery 2020

Level of Evidence $V$ This journal requires that authors assign a level of evidence to each article. For a full description of these Evidence-Based Medicine ratings, please refer to the Table of Contents or the online Instructions to Authors www.springer.com/00266.

As the report showed, 30-60\% of the Asians have a single upper eyelid or an indiscernible supratarsal fold [1]. On this condition, blepharoplasty has been the most commonly requested cosmetic operation in Asia, which is also almost synonymous with double eyelidplasty in this area. Since this technique was firstly described by Mikamo [2], it has undergone significant strides to optimize the efficiency and technical success. Meanwhile, increasing awareness of the influence of blepharoplasty on eyebrow position has been raised since it is closely related to facial expression and appearance, especially for those patients asking for blepharoplasty mainly for eyebrow ptosis.

In the article being discussed, a retrospective review of the authors' departmental case load highlighted that double eyelidplasty can lower the eyebrow in patients without a supratarsal crease which should be taken into account in surgical planning and explained to patients before surgery. The authors reviewed several studies that measured

Jie Luan

luanjieplastic@126.com

1 Department of Aesthetic and Reconstructive Breast Surgery, Plastic Surgery Hospital, Chinese Academy of Medical Sciences and Peking Union Medical College, No. 33 Ba-Da-Chu Road, Shi-Jing-Shan District, Beijing 100144, China eyebrow position after blepharoplasty with controversial conclusions. Most of these studies were limited to discrepancy of anatomical characteristics and surgical techniques.

This article has raised awareness on changes to the surrounding periorbital features, especially the eyebrow position, after double eyelidplasty. Overall, our experience mirrors that reported by this article. The eyebrow position changed in some patients, but remained unchanged in most cases in mini-incision double eyelidplasty. The relief of compensatory eyebrow elevation following double eyelidplasty may majorly account for it. For facial expression, the eyebrow is fundamental, which also establishes a person's mood. Besides, to elevate the eyebrow moving inferiorly, the frontalis muscle is recruited to result in deep horizontal forehead furrows. Moreover, the positions and shapes of eyebrow are important determinants of facial aesthetic appearance which are affected by age, sex, culture, and current fashion trends. There is great variety in the size, shape, and position of the eyebrows that are aesthetically pleasing on different faces. Though, as it measured, there was statistically significant eyebrow descent after double eyelidplasty in this paper, whether the patients perceived the change and considered it as a functional or aesthetic issue should be included in further investigation. The influence of different parts of the eyebrow shape like an arched apex and central medial part can also be analyzed precisely, the alteration of which demonstrates the facial expression.

The photographs taken before and 7 days after surgery were used to analyze the eyebrow position. Though the authors acclaimed that all the photographs were standardized with eye gaze as well as forehead and eyebrow position, the pictures in the article showed that it is hard to 
achieve. Previous techniques for eyebrow position measurement had been performed based on the relationship of the eyebrow to periorbital structures like eyelid margin or crease position that tend to change with age or are difficult to accurately measure because of landmark ambiguity [3-5]. Standard three-dimensional analysis of eyebrow shape and position based on facial bone landmarks may be a promising direction for further study [6]. The authors had a 7-day follow-up for their patient cohort which they recognized as a limitation. In our practice, the created double eyelid continues to evolve with time and is influenced by possible changes in patient age. A minimum follow-up period of 6 months is a given to evaluate and plan further surgery.

For Asian patients, eyelid anatomy, aesthetic goals, and surgical techniques are vastly different from that of Caucasian patients. As the orbital septum fuses with the levator aponeurosis well below the superior tarsal border [7-9], this lower insertion results in a fuller appearance of the upper lid or a less discernible double eyelid in the Asian population. The height of the crease also differs significantly among Caucasians and Asians with the discrepancy in the height of the tarsal plate. The tarsal height is $10-12 \mathrm{~mm}$ in Caucasians compared with $6-8 \mathrm{~mm}$ in Asians. In Caucasians, upper eyelid blepharoplasty is largely performed to handle bulky skin and preaponeurotic fat, while Asian blepharoplasty should be to create or enhance an eyelid crease consistent with the patient's ethnic appearance. Debulking of skin and preaponeurotic fat is the secondary goal for surgery. The common mistake made by surgeons is to apply Western concepts of anatomy, aesthetics goal, and surgical techniques to Asian patients. To achieve the most satisfactory result, ophthalmic surgeons should gain familiarity with the anatomy, surgical techniques as well as patients' desired outcome.
Funding None.

\section{Compliance with Ethical Standards}

Conflict of interest The authors declare that they have no conflicts of interest to disclose.

Human and Animal Rights This article does not contain any studies with human participants or animals performed by any of the authors.

Informed Consent For this type of study, formal consent is not required.

\section{References}

1. Hiraga Y (1980) The double eyelid operation and augmentation rhinoplasty in the Oriental patient. Clin Plast Surg 7(4):553-567

2. Mikamo K (1896) A technique in the double eyelid operation. J Chugaishinpo 396:9

3. Koch RJ, Pope K (2004) Quantitative assessment of brow position: a new measurement system. Plast Reconstr Surg 113:1290-1291

4. Cartwright MJ, Kurumety UR, Nelson CC et al (1994) Measurements of upper eyelid and eyebrow dimensions in healthy white individuals. Am J Ophthalmol 117:231-234

5. Matarasso A, Terino EO (1994) Forehead-brow rhytidoplasty: reassessing the goals. Plast Reconstr Surg 93:1378-1389 discussion 1390-1

6. Papageorgiou KI, Mancini R, Garneau HC et al (2012) A threedimensional construct of the aging eyebrow: the illusion of volume loss. Aesthet Surg J 32(1):46-57

7. Jeong S, Lemke BN, Dortzbach RK, Park YG, Kang HK (1999) The Asian upper eyelid: an anatomical study with comparison to the Caucasian eyelid. Arch Ophthalmol 117(7):907-912

8. Haramoto U, Kubo T, Tamatani M, Hosokawa MK (2001) Anatomic study of the insertions of the levator aponeurosis and Müller's muscle in oriental eyelids. Ann Plast Surg 47(5):528-533

9. Carter SR, Seiff SR, Grant PE, Vigneron DB (1998) The Asian lower eye-lid: a comparative anatomic study using high-resolution magnetic resonance imaging. Ophthalmic Plast Reconstr Surg 14(4):227-234

Publisher's Note Springer Nature remains neutral with regard to jurisdictional claims in published maps and institutional affiliations. 